\title{
Supporting the Creative Game Design Process with Exertion Cards
}

\author{
Florian 'Floyd' Mueller' \\ ${ }^{1}$ Exertion Games Lab \\ RMIT University \\ Melbourne, Australia \\ Martin R. Gibbs ${ }^{2}$ \\ Frank Vetere ${ }^{2}$ \\ ${ }^{2}$ Computing \& Information Systems \\ University of Melbourne \\ Darren Edge \\ Melbourne, Australia \\ ${ }^{3}$ Microsoft Research \\ Beijing \\ China \\ floyd@exertiongameslab.org, \{martin.gibbs, f.vetere\}unimelb.edu.au, darren.edge@microsoft.com
}

\begin{abstract}
Advances in sensing technologies have led to research into exertion games that support physically effortful experiences. Despite the existence of theoretical frameworks that can be used to analyze such exertion experiences, there are few tools to support the hands-on practice of exertion game design. To address this, we present a set of design cards based on the "Exertion Framework", grounded in our experience of creating exertion games for over a decade. We present results demonstrating the value and utility of these Exertion Cards based on our studies of their use in three workshops held over seven sessions with 134 design students and experts. We also articulate lessons learned from transforming a theoretical framework into a design tool that aims to support designers in their practice.
\end{abstract}

\section{Author Keywords}

Exergame; whole-body interaction; exertion interface; creative process; design cards; workshops; game design

ACM Classification Keywords: H.5.2. [Information Interfaces and Presentation]: User Interfaces Miscellaneous.

\section{INTRODUCTION}

There has been a recent trend in HCI that places the human body at the center of the user experience, fostering "exertion"-based interactions that require intense physical effort from users [18]. Computer games currently provide a growing range of exertion systems, with the Microsoft Kinect, Nintendo Wii and Sony Move, along with virtual

Permission to make digital or hard copies of all or part of this work for personal or classroom use is granted without fee provided that copies are not made or distributed for profit or commercial advantage and that copies bear this notice and the full citation on the first page. Copyrights for components of this work owned by others than the author(s) must be honored. Abstracting with credit is permitted. To copy otherwise, or republish, to post on servers or to redistribute to lists, requires prior specific permission and/or a fee. Request permissions

from Permissions@acm.org.

CHI 2014, April 26 - May 01 2014, Toronto, ON, Canada. Copyright is held by the owner/author(s). Publication rights licensed to ACM.

ACM 978-1-4503-2473-1/14/04 ..\$15.00.

httb://dx.doi.org/10.1145/2556288.2557272 reality exercise bikes [16], mobile games fueled by exercise $[5,14]$ and our own systems $[17,18,20,21]$ populating a design space that highlights the potential of such mediated exertion experiences. Researching these games is important, as they can offer mental and social benefits, as well as benefits to physical health [13]. Research has previously highlighted a limited theoretical understanding of these exertion experiences [7, 9]. In response, we developed the "Exertion Framework" [19] - a systematic conceptual understanding of the ways in which the body engages in exertion experiences as well as the ways in which interactive technologies can support such experiences.

However, our Exertion Framework, like so many other HCI frameworks [11], can be criticized for not offering "stepby-step guidance" for designers and hence not lending itself to support the designer's creative process [11]. We agree with Hornecker when she says that "frameworks tend to be systematic and abstract, which makes them hard to use in creative practice" [11], and the Exertion Framework can probably also be characterized by this abstract nature. Despite frameworks offering designers the ability to reason and justify design decisions, the gap between theoretical frameworks and the practical design process has long been an issue for HCI and a source of frustration for many practice-based design researchers $[1,6,12]$. Recent research has investigated how to close this gap in order to support design researchers $[1,11]$, with one suggested way being to turn the framework under investigation into a design tool [11]. In particular, Hornecker proposes to transform the abstract themes of a framework into colloquial questions to which designers can relate [11]. These questions are then framed in a card-based format and introduced to the design process in order to offer guidance from the themes of the framework. As evidence for the utility of this approach, she demonstrated the transformation of a tangible framework into design cards and used them in a series of workshops with positive outcomes in the form of novel design ideas [11].

We utilized a very similar approach to transform our abstract framework into a useful design tool. We opted to lean on this prior work as the conditions are comparable: the author, like us, devised her own framework, which is 
based on an embodied approach to interaction design [11], very similar to our investigations into exertion. Furthermore, both her and our work is targeted at designers of interactive technology.

More generally, our research builds on previous work on design cards and associated workshops [2, 8, 10, 15, 23]. Physical cards used to facilitate design activities have previously been shown to function as orienting devices, providing structure to the creative sessions and supporting progression from the initial starting point [11]. The physicality of the cards can help make arguments tangible during discussions, supporting focus and helping create common ground [4]. Furthermore, design cards support focus shifts, as they can be spread out and distributed amongst participants [10]. Previous research on the use of design cards for game design has also been shown to facilitate the ideation process $[8,15]$. We built on this prior work and transformed the Exertion Framework into a set of design cards. As a result, our work is the first that presents design cards specific to exertion games. Our work is also the first that explicitly encourages a consideration of tradeoffs among the dimensions articulated with the design cards, extending the notion of trade-off-driven design from Cognitive Dimensions (for example see [3]) to design cards. Lastly, we also examined how these design cards supported the ideation process in three workshops held over seven sessions with 134 participants. With this, we are able to provide details on the use of individual cards and insights on each card's role in the design process as well as detailed challenges when designing with cards, extending prior design card work that mostly offers a coarse view on people's experiences with using the broad themes articulated by a range of cards. With this approach, we aim to offer designers of future cards structured guidance on the design of each individual card.

\section{THE EXERTION FRAMEWORK}

The Exertion Framework [19] consists of four lenses through which exertion can be examined. These lenses, conceptualized as concentric, onion-like layers, offer different perspectives through which to examine a set of themes, or "things to think about". The lenses are: The Responding Body (how the body's internal state changes over time as a result of exertion, e.g. heart rate), the Moving Body (how body parts are muscularly repositioned relative to one another), the Sensing Body (how the body is sensing and experiencing the world) and the Relating Body (how bodies and people relate to one another). These four lenses provide different perspectives on each of six cross-cutting exertion themes: uncertainty of exertion; awareness of exertion; expression of exertion; rhythm of exertion; risk of exertion; and understanding of exertion [19].

\section{Turning the framework into design cards}

Prior work presented the usefulness of transforming a framework into design cards, but does not describe a process of how to turn a framework into design cards [11].
We therefore constructed our own process, but we were guided by her examples as well as our own experiences of using the framework in our practice of designing exertion games for over a decade. The goal was to turn the framework's themes into colloquial, provocative questions whose purpose is to promote creative design thinking.

We also added a "dimension" to each question, which highlights that interactive qualities are not simply present or absent, but can vary in their degree from a little to a lot. For example, "uncertainty of exertion" can be supported "a little" or "a lot". Furthermore, a dimension highlights also that choosing to keep a theme at a minimum (e.g. by preventing it from occurring) is also a design decision that can have merit.

We now describe the process we created to turn the themes of the Exertion Framework into design cards. This process is not the only way to turn themes into cards, nor has it been validated beyond our own work. Nevertheless, we structure it via groupings that we believe might be useful for others when orienting themselves to a similar task. With this approach, we aim to extend prior work on design cards that often tends to focus on the design of theory as cards (for example see [11]) by providing one of the first attempts to detail the transformation of design theory into cards. Our process consisted of the following five stages:

\section{Establish target boundaries}

We began by establishing target boundaries, in particular, we decided to aim for a relatively small number of cards (ultimately 14) in order to minimize the chances of designers feeling overwhelmed.

\section{Scrutinize framework}

We looked at the themes of the Exertion Framework, and picked one aspect of each that related to one particular layer of the body lens. For example, for "rhythm of exertion", we selected the "Relating Body". Furthermore, we also picked each lens individually to support the analysis of technology augmentation. For example, we transformed the "Responding Body" into a question about (measuring) effort.

\section{Reduce items}

In order to reach our target boundary, we focused on questions we felt could challenge game designers. This resulted in 14 questions.

\section{Visualize}

We wrote the questions on index cards and gave each design card a short and descriptive title. We also selected two pictures to represent examples of either end of the dimension. We used images that offered easy entry points into the topic while at the same time not implying any specific use. Next, we assigned a positive example to each end of the dimension to provoke the reader to consider divergent possibilities, so that a reader might ponder: "If I support a lot of this, I will foster experience $\mathrm{X}$, but if I support a little, I will foster experience $Y$ " (where $Y$ is not simply the absence of $\mathrm{X}$ ). For three cards, we opted to 
provide additional details to clarify what it would mean for participants if they would choose the middle of a dimension that has no obvious medium value: for example, we highlighted that medium intensity can mean that intensity in movement might not be interpreted by the system, but could be communicated to another person.

\section{Incorporate feedback}

After having generated all cards, we discussed the results extensively with senior researchers who gave feedback on how to improve the cards' wording and their presentation. Based on this feedback, we refined the associated examples and the card layouts several times. In addition, we discussed the cards with two fellow researchers in order to verify that the descriptions on the cards were understood even if we were not explaining them.

The cards were color coded with four different colors, one for each lens of the framework (Fig. 1). The centerpiece of each card was the question that came out of the transformation process above. These questions were meant to work on a more pragmatic level than the theoretical concepts [11]. We aimed to make the questions easily accessible in order to support the creative process rather than requiring a deep understanding of the framework. The challenge was to find a balance between abstract concepts and inspiring questions, without falling into the trap of presenting rules that suggested they needed to be followed [11]. The aim was to create questions that designers use as thought-provoking suggestions to guide their creative process. The cards are available at exertiongameslab.org.
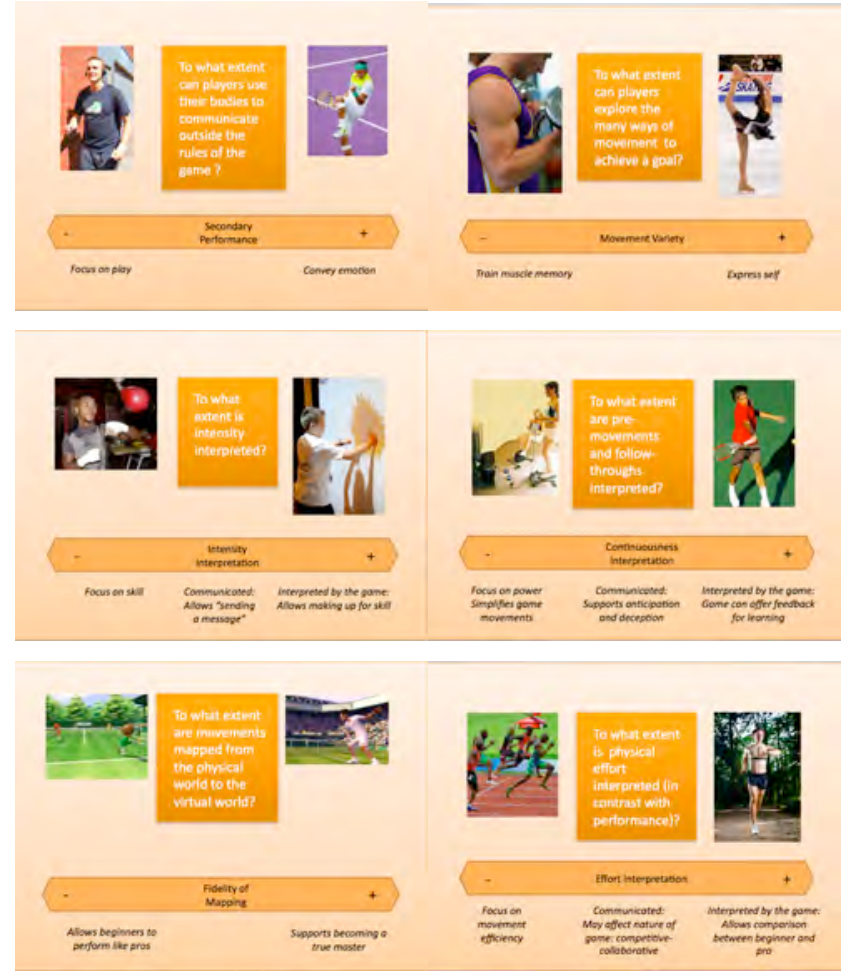
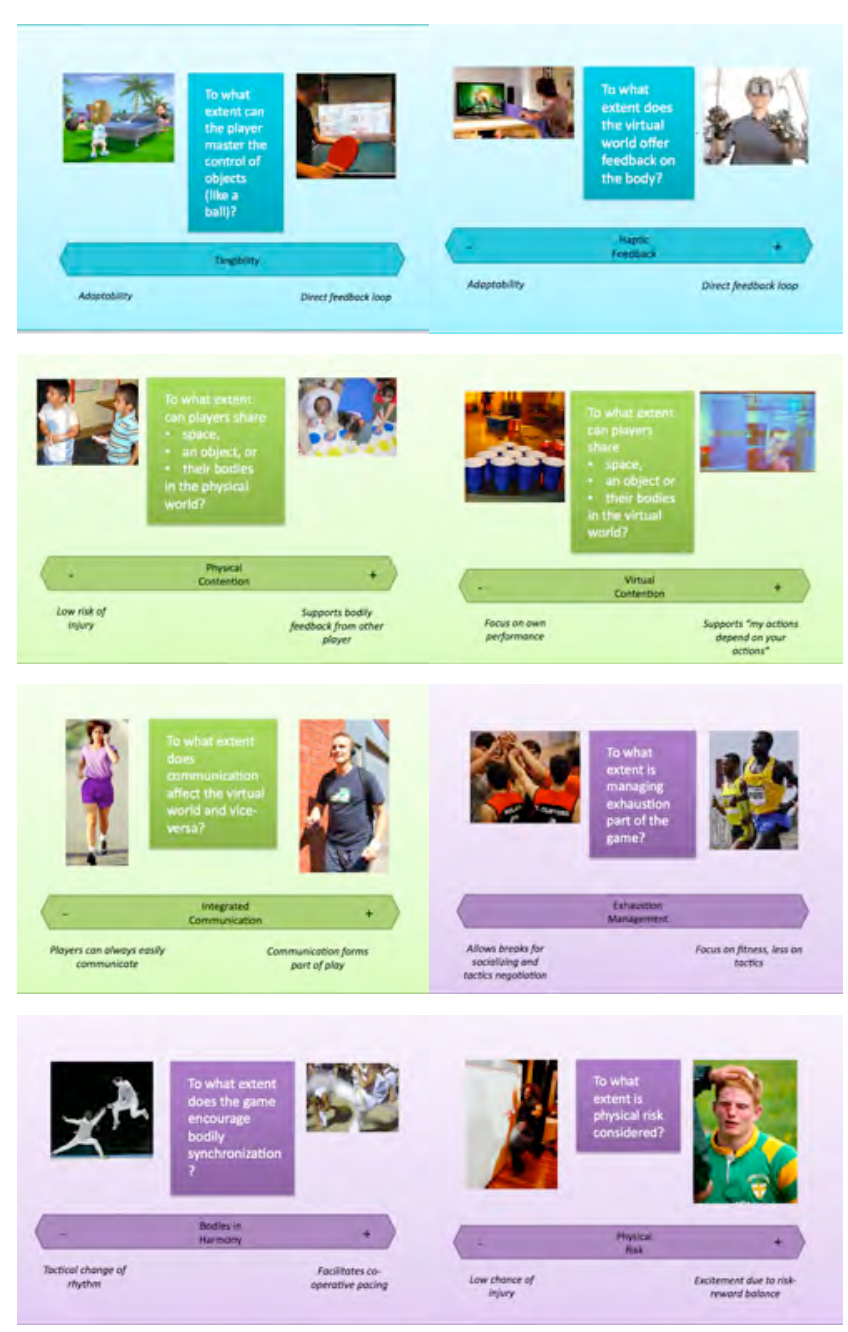

Figure 1. The Exertion Cards based on the Exertion Framework

\section{WORKSHOPS}

We decided to study the use of the design cards in workshops with designers to see how they could be incorporated into the creative design process. The purpose of this study was to demonstrate the value and utility of the design cards in designers' practice, which in turn would strengthen the argument for the usefulness of the framework. Evaluating the design cards in short (3h) workshops is of course not the same as a designer's day-today practice. However, previous work has also utilized workshops $[8,11,15]$, suggesting that they might be a way to approximate design practice. Furthermore, a workshop with team-based design exercises is similar to the teamwork environment to which designers are often exposed. Lastly, workshops provide a tight, time-constrained format for creative teamwork, which we found similar to the environments we have encountered in our own practice.

\section{The Workshops}

We conducted three workshops, with two of them conducted over multiple sessions to accommodate large participant numbers. Workshop $\mathrm{A}$ and $\mathrm{B}$ were repeated 
twice and four times respectively (Table 1). The workshops were carried out across three sites, with sessions adapted to the different contexts, number of participants and room layouts. Each participant attended one session only.

\begin{tabular}{lllll}
\hline Workshop & \multicolumn{2}{l}{ Participants (in sessions) } & \multicolumn{2}{l}{ Context, Duration } \\
\hline A x 2 & $\begin{array}{l}\mathbf{1 4} \\
(8,6)\end{array}$ & $\begin{array}{l}\text { Designers \& } \\
\text { researchers from } \\
\text { two technology } \\
\text { companies }\end{array}$ & $\begin{array}{l}\text { Conference } \\
\text { room }\end{array}$ & h \\
B x 4 & $\mathbf{5 5}$ & Design students & $\begin{array}{l}\text { Design } \\
\text { studio }\end{array}$ & $3 \mathrm{~h}$ \\
& $\begin{array}{l}(14,14, \\
13,14)\end{array}$ & Classroom & $3 \mathrm{~h}$ \\
C x 1 & $\mathbf{6 5}(65)$ & $\begin{array}{l}\text { Digital game } \\
\text { design students }\end{array}$ & \\
\hline Total & $\mathbf{1 3 4}$ & & & \\
\hline
\end{tabular}

Table 1. Three workshops across seven sessions with 134 participants in total.

\section{Structure of the workshops}

The three workshops had different constraints and opportunities but all shared the same structure around the cards (Fig. 2). The participants were invited with the premise they would learn about designing exertion games, and as an exercise they were asked to come up with an exertion game idea. We began each workshop by defining the term exertion game ("a digital game that requires physical effort from players" [19]). We then showed video examples of exertion games before explaining the design challenge. The goal of the workshops was for each team to design an exertion game. We did not want to confine the scope of the workshop too much, so participants could make the content applicable to their respective design practices. For example, the design students could work on a game idea that they might want to develop further in their classes.

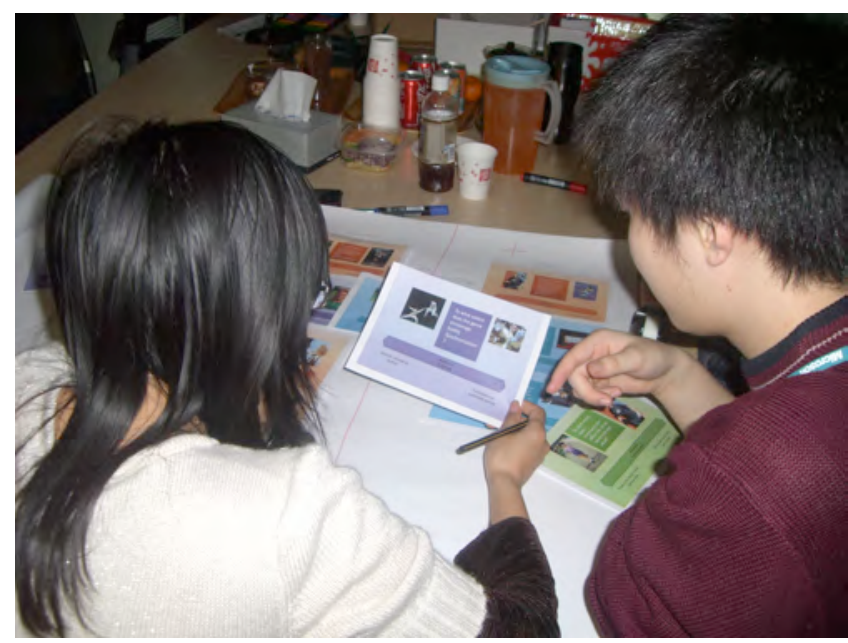

Figure 2. Participants discussing a particular design card

\section{Participants}

In Workshop A, the 14 participants were volunteers from Microsoft Research in Beijing, China (Microsoft Research was working on the Kinect at the time). The participants' backgrounds ranged from Computer Science to HCI and Design, which is representative of the target group of the design cards. The second session was conducted in the same company, but for this session, half of the participants came from Nokia Research's team that was interested in supporting their customers' play desires. In Workshop B, 55 design students took part across four sessions that were conducted in a design studio in Australia. In workshop C, 65 students from a game design course in Australia participated in a classroom setting. This setting was less adaptable to group work, but participants were free to roam during the workshop.

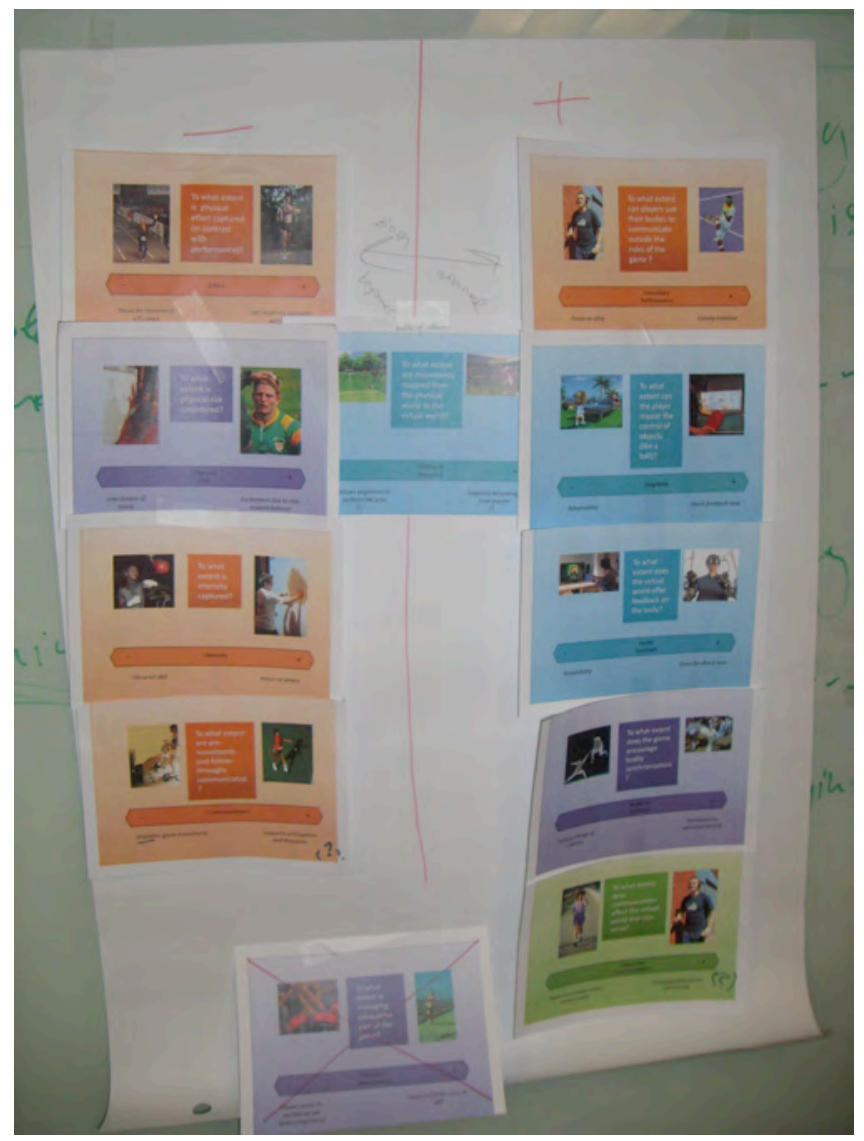

Figure 3. The participants placed how they used the cards on a dimension from "a little" to "a lot". The card on the bottom indicated that it was "not useful"

\section{Task}

Each workshop lasted 3 hours. The participants were asked to form teams of around 5 people. Most sessions had teams of 4-5 members, with the remaining participants (due to the total amount of participants) forming smaller teams down to 2 members. The challenge was to design an exertion game. The goal was to come up with an original idea that participants were asked to present to their peers at the end of the workshop. Each team was asked to write down or 
sketch out any game ideas they might have. They were free to explore if and how the design dimensions could support them in their creative process. Once a team decided on a final design, and there was time due to the other teams still working, they were asked to mark on a large sheet of paper which design dimensions (if any) they used and how. We suggested they align the cards along a spectrum from positive to negative, with neutral in the middle. For example, if the group decided to incorporate "a lot" of "integrated communication", they attached the card to the "+" side of the paper, if they decided to include only "a little", they attached it to the "-“" side. Participants were also encouraged to discard cards if they did not think they were useful for their particular task (Fig. 3).

\section{Feedback}

After the exercise, the participants were interviewed and their answers recorded. The posters they used to present their ideas and any other supporting materials they developed were also captured. Due to the size of workshop $\mathrm{C}$, not all participants had a chance to provide feedback; as a result, those participants were also given the opportunity to post any comments on a dedicated website. Participants of all workshops were also given a questionnaire at the end that asked them about each card, including how useful they thought it was. Furthermore, participants were asked what their favorite card was and why. This question was designed to reveal how cards could be made most applicable to their design practice.

\section{Data collection}

We captured video for the entire participant group for each workshop. After each design exercise, the participants were interviewed per team, and their answers were video recorded per team and notes were taken. The final presentations of each team were videotaped, and any sketch and note materials were photographed. The comments on the website were captured and analyzed, as were the questionnaires. We were also interested if and how participants used the cards beyond the workshop, and therefore interviewed the participants of the workshop $\mathrm{C}$ two months after their workshop. 75 participants of the original workshop were available for this group interview.

\section{Data analysis}

We initiated the analysis by reading and watching all the material. Then we started the coding of the data, guided by the processes suggested by Strauss and Corbin [25]. We began by looking for all relevant mentions of design cards. This meant identifying instances that described if and how design cards were used. We also looked for any other instances that related to the cards and any themes from the framework. This included looking for evidence that pointed to the use of the cards in a way that sparked new ideas or changed existing ones. In addition, the data from the questionnaires was analyzed and visualized using a spreadsheet program. In session 2 of the first workshop we accidentally did not include the contention cards in the questionnaire. As a result, we did not generate graph plots of these two cards from the questionnaire data.

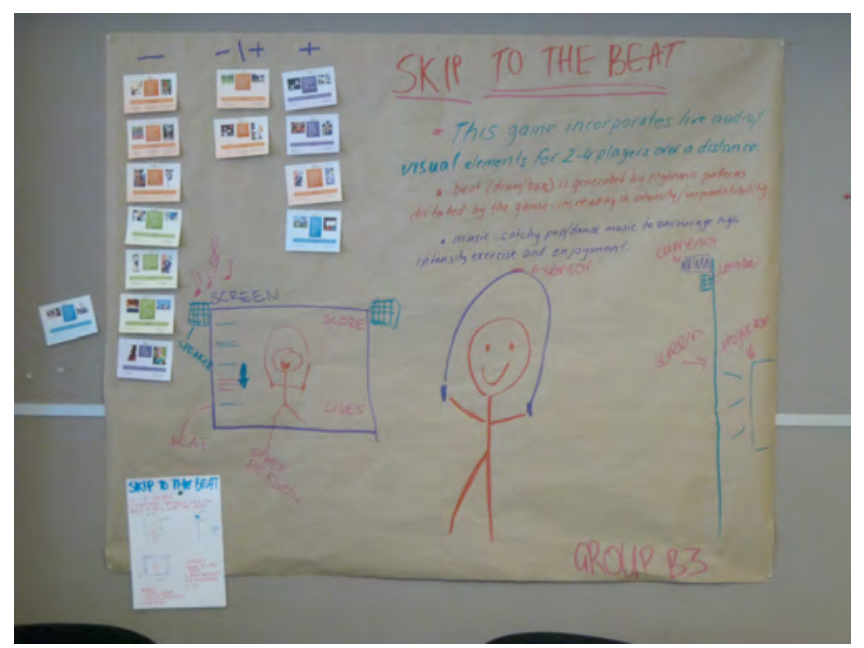

Figure 4. Skip to the Beat: augmented jump rope promoting multi-player skipping to music beats.

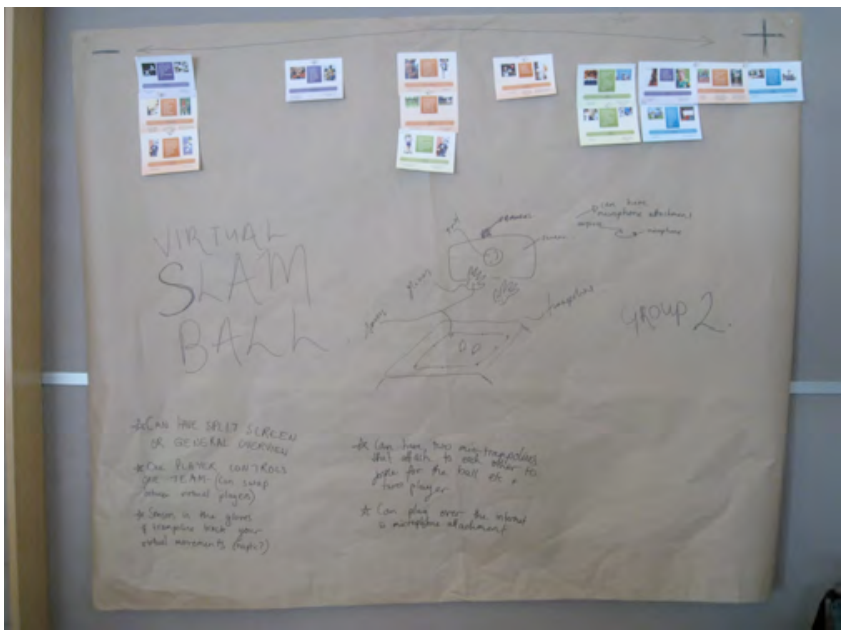

Figure 5. Virtual Slam Ball: 2-player slam ball experience with sensors in gloves and trampoline

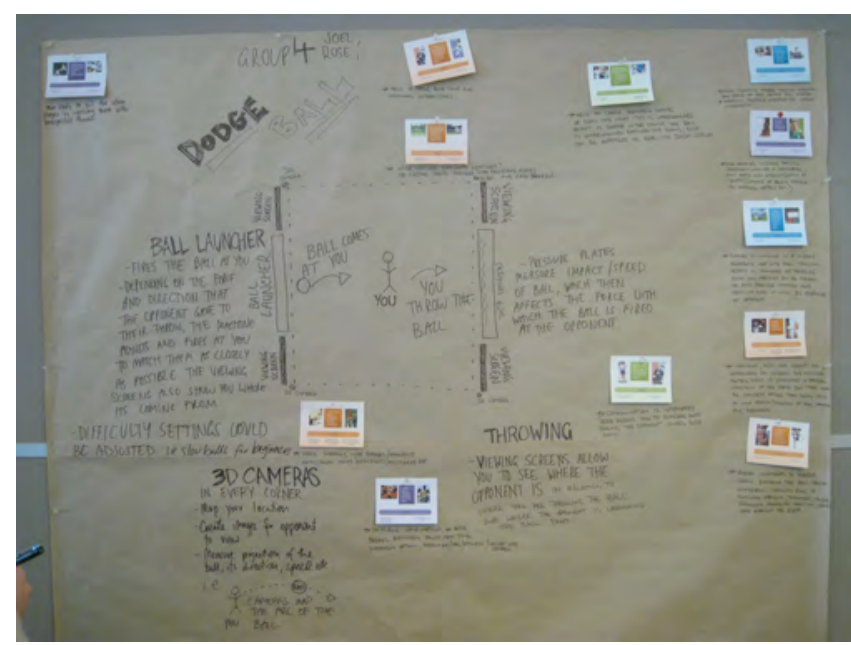

Figure 6. Dodge ball: augmented gym that tracks and throws balls for larger group exertion experiences 


\section{RESULTS}

First, we present a few of the ideas participants came up with during the sessions in order to suggest that the workshops led to creative ideas. The pictures are photographs of the posters presented, with design cards attached by participants according to their use (Fig. 4, 5, 6). Next, we will elaborate on the role of the cards on the creative process that led to these ideas.

\section{Perceived usefulness of cards}

The data from the questionnaires suggests that participants agreed with the statement that each card was useful for their design task. In particular, a majority of participants either agreed or strongly agreed to this statement in the questionnaire (Fig. 7).

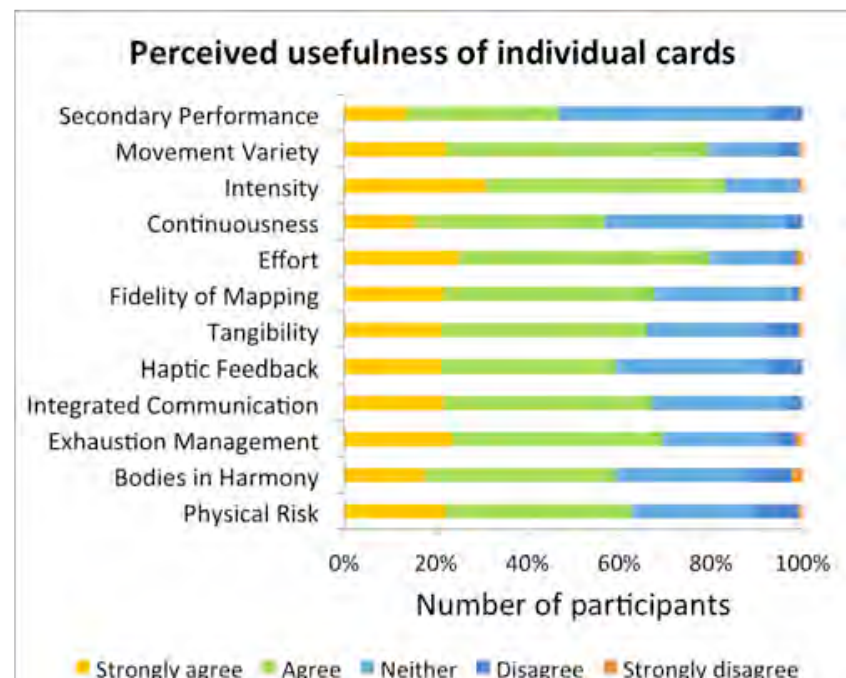

Figure 7. Likert-scale results on how useful participants found each card

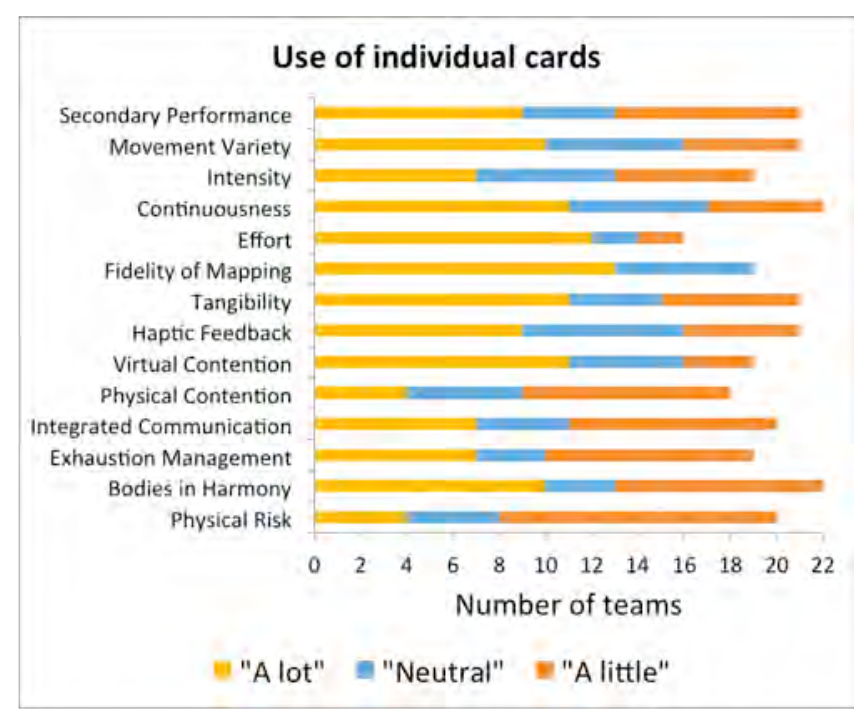

Figure 8. The placement of the cards in terms of use.

\section{Use of individual cards}

The next figures display how participants' teams used the cards for their particular design task. A typical placement of the design cards can be seen in Fig. 3. When asked to place their cards on a dimension from "a little" to "a lot" in response to how they used a particular card, the following data resulted (Fig. 8).

Participants were also asked to note or discard the cards they did not find useful (see Fig. 9). There was no card that all teams found "not useful"; this suggests that every card was useful for at least one team. Out of 22 teams who placed their cards, six teams (the highest number) found "effort interpretation" not useful.

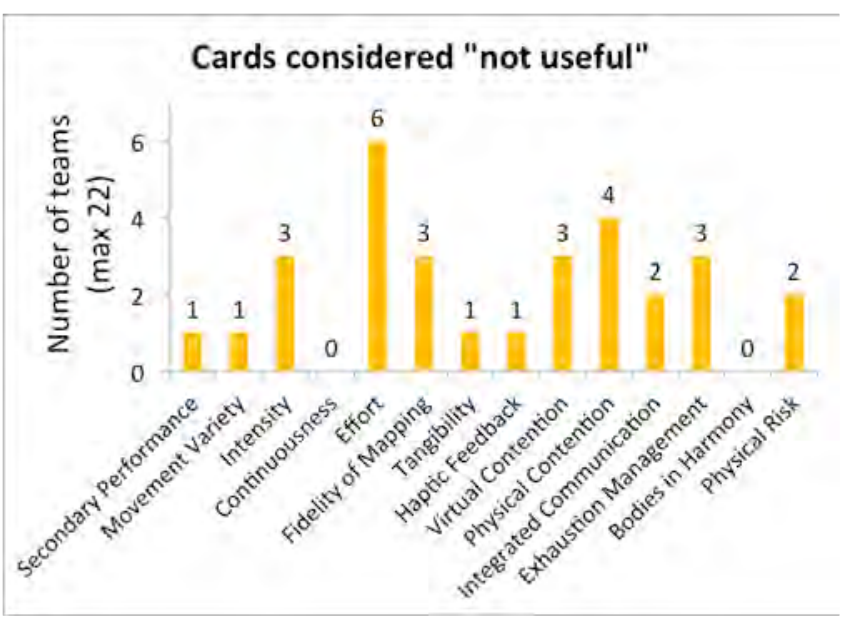

Figure 9. Cards not placed on the dimension

The majority of teams found only one to two cards not useful. The highest number of cards one team did not consider of value was 9 (see Fig. 10).

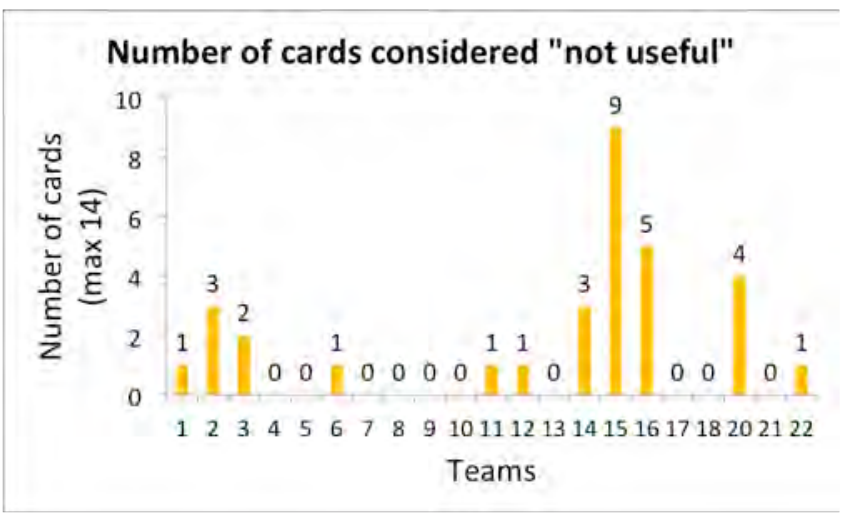

Figure 10. The amount of cards not placed on the dimension.

\section{Role of cards}

The participants were also asked at the end of the workshop what role the cards played in their design process. The data show that the cards had multiple roles. When combining "Strongly agree" and "Agree", the following list emerges with the top answer listed first. The individual composition can be seen in fig. 11 . 
1. The cards helped me to improve my ideas

2. I found the cards useful for generating ideas

3. The cards helped my team to focus

4. The cards helped me to articulate my ideas

5. The cards helped my team to come to a consensus/agree on items we discussed

6. I had ideas I would not have had without the cards

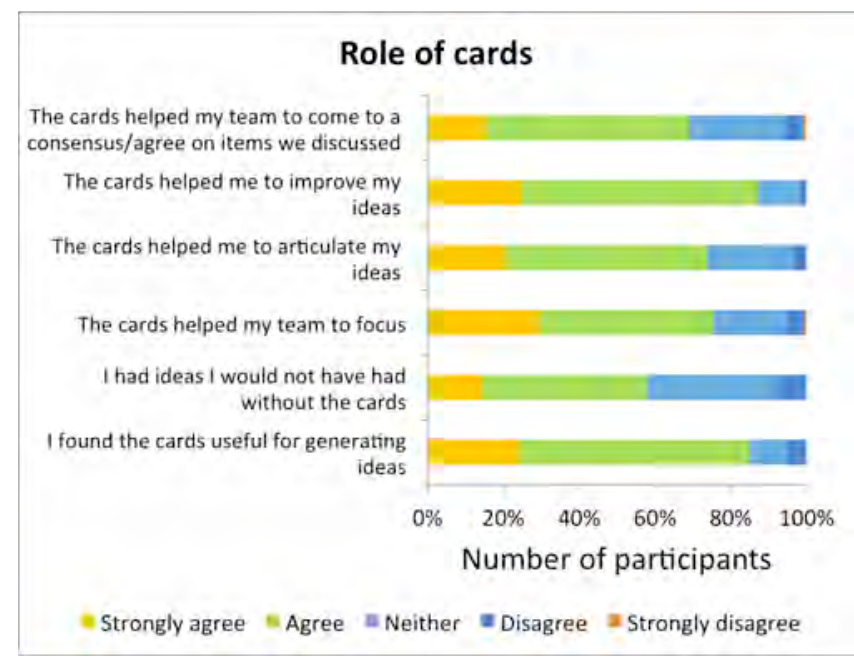

Figure 11. How participants assessed the role of cards

Through a freeform field, the questionnaire gave participants the option to note any additional roles they thought the cards played. 59 participants thought the cards had additional roles. By structuring them based on logical closeness, we derived three major additional roles. The cards helped participants to fine-tune their ideas, offered guidance for the design process, and supported expanding participants' horizons.

\section{Cards helped fine-tune ideas}

Twelve participants stated that an additional use of the cards was to fine-tune their ideas. They expressed that the cards supported them in making their idea more concrete and explicitly focused on the experience they wanted to create. A common statement was: "The cards helped refine our ideas."

\section{Cards offered guidance}

Six participants found that the cards offered them some guidance as a group on how to proceed with the design task: "They are good guidelines/building blocks." "It gave our group clear direction and order, something that we sometimes lack." The cards provided a "framework for analysis."

\section{Cards expanded horizons}

A strong theme was also that the cards helped to expand participants' horizon: "It enabled our group to consider areas that we normally would not consider, like synchronization." This resonates with another statement that appeared repeatedly: participants appreciated that the cards helped them to consider "all aspects" of the game, reminding them of perspectives that they otherwise might have overlooked: "[The cards helped] generating and incorporating ideas that weren't thought of before."

\section{Danger of card reliance}

One participant was critical in his/her comment, saying that the cards have the potential to overcomplicate the process: "They gave a broader outlook of possibilities, however not necessarily 'better' ideas. In perspective of 'easy fun'/'hard fun' stuff, some cards may overcomplicate games for a particular audience."

\section{Additional results}

The data coming from the video recordings and freeform fields provided additional insights detailed below.

\section{Offering guidance}

The cards offered guidance to the creative process. The participants called the design dimensions an "easy to understand guideline for starting and refining games." Others found that the cards helped them identify aspects of their design they had overlooked so far, further strengthening the view of the cards as a guide: "I think the dimensions for exertion games work really well to help guide the design of a game. While our group discussed each card in reference to our game I found the dimensions helped us to work out what our game might be lacking, and what could be added to improve it." Some participants saw the cards as tools that not only guided existing ideas, but also helped generate new ones: "The flashcards are very useful for people such as myself, because I can never come up with an idea on the spot. The dimensions are perfect for producing further ideas to elaborate on as well as a guide." In this sense, people used it as a tool: "the idea of game design [is now] a whole lot deeper. Like most I found the design dimension cards to be a useful tool in improving a game."

One participant praised the potential of the cards for industry and academia alike: "I think that as a tool, not only to be used by game companies but also for teaching, the design dimensions are really great." Another participant noted how the design dimensions will be useful as a guide for future games and how they will be used in his/her practice: "I found the design dimension cards to be a useful guideline. [...] I will be referring to it from time to time, in order to analyze the quality of our next game."

The cards offered the participants a more efficient way to reach their goals when compared to their existing methods. They praised how they generated results much quicker when using the design dimensions: "I found the dimensions really helpful, by being aware of the elements that are in games, it makes it so much easier, faster and most likely better to come up with new games and improve existing ones. I found dimensions jump-started my thoughts in a way, that most cards got me thinking of new ideas and/or ways to improve them and got me thinking about different 
aspects of games that I had not considered before and now seem integral." "We obtained a lot of fresh ideas and faster than any other method I know myself. But to say that we would have never gotten these ideas without them is another thing, I think it's a time factor more than a possibility factor."

\section{Shifting processes}

The cards helped the participants to see how they could approach the creative task in a different way. They described the use of the cards "as an eye opener", helping to "view design in a different way", as "the idea of dimensions helps me think."

One participant explained how the cards helped the group "to rediscover what it is specifically that we want out of our game. It has given us a ground, or a foundation, from which we can mold and develop our concept". By having a clear goal, participants found it easier to assess their ideas: "They're a really useful and clear way of looking at our games for refining and moving beyond 'What can we add to our game?' to 'What could we add to our game to emphasize or draw out more of a certain quality our game has or needs?'"

\section{Factorizing tasks}

The cards helped some participants break down the creative process into individual elements that were easier to handle. Firstly, the cards enabled them to identify that there are several elements that make a successful game: "[it made me] realize that so many factors can affect the game and make it different."

Secondly, the cards helped participants to identify these different elements. In other words, the cards supported them in breaking down the task into individual factors: "I did like the break down of the categories." "Say you have a job to do [...] The dimensions would help you define the different aspects of the game and what kind of rules and gameplay to include."

Equipped with these factors, many participants felt empowered to make strategic decisions during the design task: "What made [altering the game] easier was that the cards gave definable characteristics of exertion games, so rather than thinking 'how do I make it more physical?' we could pin point several aspects of the game and the 'player experience', and alter one factor, rather than trying to pull something new out of thin air and risk changing everything."

\section{Weighing up options}

The cards allowed some participants to assign a qualitative weight to certain aspects of their designs: "The cards were really helpful in figuring out where our game's strong points are, or more importantly where they are not." Being able to weigh up certain elements gave many participants the confidence to focus on specifics, which they used to identify which aspects to improve first: "It makes us know what we need to do for improving our game." "I found the cards to be incredibly helpful, especially in regards to tweaking some flaws in our game idea."

\section{Focusing the aim}

The cards helped some participants focus on the main aim; creating an engaging game. "This sort of thing is great for someone like me who tends to get caught up in what they're focusing on and slowly becoming more and more tunnel visioned." The cards helped them to stay true to the original goal of making an engaging game by diverting them from any distractions such as attention to technical implementation too early: "I believe the dimensions were a great way of allowing us designers to refocus on the essence of the game; the approach to 'fun' if you will. [Without the cards] we tend to concentrate too much on technology and implementation, and less on how the player(s) are enjoying the game. “

\section{DISCUSSION}

We now discuss the individual findings in detail, relating them back to the cards and the framework. In summary, byand-large, the cards were found to be useful in the creative design process.

\section{Usefulness}

The majority of participants considered all cards useful in their design task. They found the cards useful as a whole, and most participants did not differ much in their assessment of individual cards. The following sections discuss how participants used the cards during the design process.

\section{All cards were useful}

Participants used many cards "a lot" or "a little" as indicated by the placement task, and found only a few not useful for their particular design process. No single card was considered "not useful" by all teams, suggesting that each card had found a use in the workshops. This indicates that teams found the cards useful. As expected, not all cards were useful for all design processes, but participants found more cards useful than not useful. "Effort interpretation" and possibly "physical contention" were the cards that received the most (although still modest) count of "not useful", suggesting that in future iterations of the cards, these cards should be refined first.

\section{What cards were useful for}

Participants confirmed that the cards were useful for idea generation, idea improvement and articulation. The cards also offered a language to discuss certain aspects of the design, and supported teams in focusing their efforts. In addition to these anticipated roles, the participants also expressed that the cards helped them to fine-tune existing ideas, and helped them to be more concise in their efforts. The participants also appreciated the cards as a guide and found their role in the creative process valuable in terms of goal development, efficiency and effectiveness. In sum, the 
cards offered a structure for the design process that often expanded their range of ideas.

Such an assessment of the roles of the cards is similar to previous findings [11]. The data suggest that the design cards do not fulfill an isolated role in the design process, but can rather serve multiple roles and complement other methods, depending on the way participants see fit for them.

The cards also helped (re-)focus participants' aim towards the "bigger picture" of creating an engaging game, and diverted them from getting bogged down in details too early. The cards were simultaneously able to support the identification of individual elements (such as technical implementation details) while highlighting the bigger picture (such as the desired play experience). So while the cards supported a micro-level view on the task at hand, they also brought a macro-level to the participants' attention.

\section{Challenges when working with cards}

The study also revealed that turning conceptual frameworks into practical design guides it not an easy undertaking. Participants valued the benefits the cards can provide, however, it was important for them that the tool does not get in the way of the creative process. For example, some participants criticized that the wording of the design dimensions was sometimes difficult to interpret, and that some cards were too similar to others. In particular, a few participants expressed difficulty in understanding the meaning of some cards, noting that the study was undertaken within different cultural contexts and with participants who had different first languages: "I found myself rereading some cards because I found them slightly hard to work out." When cards were difficult to comprehend, participants' focus shifted from the creative process to interpreting the cards, reducing the benefits of the cards. Future revisions of the cards should refine the wording and images for easier comprehension. Another solution could be to reduce the number of cards, although many participants were generally in favor of the amount of cards: "The amount of cards were just right to grab just about every aspect of a game".

\section{Supplementing other methods}

As mentioned in prior work [11], the cards are not a method: the designers still have to sort the cards themselves and find out in which order (if any) they apply them (and which cards to discard). The purpose is to facilitate but not restrict the creative process. However, some participants expected more directive guidance from the cards. This may have been due to two reasons: a) participants wanted a method as they felt this could enhance their creative process; and/or b) participants expected a method from the instructions given and were disappointed that they did not receive one.

\section{LIMITATIONS}

As with any investigation that aims to combine theory with practice, there are limitations that are shaped by both theoretical and practical concerns. One limitation of the work is that it was us who presented the design cards during the workshops, adding potentially our own personal bias. However, two of the sessions in workshop B were run by other interaction designers. Although they were instructed by us, this suggests that the design cards can be used in other settings where we are not the main facilitator, indicating that the knowledge lies in the design cards, not in the person presenting them.

A further limitation of the work is that we did not attempt to evaluate the quality of the ideas generated. We find assessing the quality of creative work challenging, we therefore opted to focus on how the design cards facilitated the creative process, rather than on judging the quality of the generated ideas. To hint at the creative contribution the participants made in the workshops we included a snapshot of some of the ideas in the form of the posters generated that suggest that the design cards can indeed facilitate creative ideas. On the other hand, we acknowledge that assessing the quality of the ideas (for example through processes such as suggested by [24]) might add weight to the argument of the usefulness of the design cards.

Furthermore, comparing the results from our workshops is limited by the fact that our participants had different expertise levels, consisting of both researchers and students. However, this approach allowed us to examine the use of the cards across a range of early career to more senior designers.

Lastly, another limitation was the use of a questionnaire. Questionnaires can only paint a limited picture of a complex phenomenon, such as the design task investigated in this study. However, we wanted to give all participants the opportunity to provide feedback, and considering the number of participants in some of the workshops, a questionnaire seemed a practical tool to achieve this. In order to minimize the limitations of questionnaires [22], we always looked for confirming statements for each questionnaire entry in the interview data.

\section{CONCLUSION}

The exertion design cards were considered useful by participants in design workshops for the creative task of designing an exertion game. The participants expressed that the cards, which were derived from the Exertion Framework, supported them in their task, suggesting the utility of the design cards and in turn the usefulness of the framework. The cards achieved this by, for example, helping the participants break-down the task into individual elements. This resulted in participants realizing that they might have overlooked an important aspect if they had not used a specific card. 
This work therefore extends prior research by providing further evidence to the value of transforming frameworks into design tools. In particular, this work makes a contribution by presenting the Exertion Cards as well as a description of the process that lead to them based on the Exertion Framework. Furthermore, this work makes a contribution by demonstrating the utility of the design cards by adding empirical evidence that the design cards can positively affect the design process of exertion games: the participants found that the design cards covered (almost) the entire spectrum of themes they found valuable for their practice, and considered the amount of cards appropriate. This adds weight to the argument that the framework consists of the major themes relevant for exertion games.

In sum, the study demonstrated the Exertion Framework in action through a set of design cards. The data collected from a range of workshops with an extensive number of participants indicates that the design cards can support the creative process of designing exertion games.

\section{ACKNOWLEDGEMENTS}

We thank Bert Bongers and team, Microsoft Research and Nokia in Beijing. Florian 'Floyd' Mueller is the recipient of an Australian Research Council Fellowship (110101304).

\section{REFERENCES}

1. Antle, A.N. Embodied child computer interaction: why embodiment matters. ACM Interactions, March + April. (2009), 27-30.

2. Belman, J., Flanagan, M. and Nissenbaum, H. Instructional Methods and Curricula for "Values Conscious Design". Loading... 3 (4). (2009).

3. Blackwell, A. and Green, J. Notational systems - the Cognitive Dimensions of Notations framework. in Carroll, J.M. ed. HCI Models, Theories and Frameworks: Toward a multidisciplinary science, Morgan Kaufmann, 2003, 103-134.

4. Brandt, E. and Messeter, J., Facilitating collaboration through design games. In Proc. Participatory design: Artful integration: interweaving media, materials and practices-Volume 1, (2004), ACM, 121-131.

5. Consolvo, S., Everitt, K., Smith, I. and Landay, J.A., Design requirements for technologies that encourage physical activity. In Proc. CHI 2006, ACM Press (2006), 457-466.

6. Dan R. Olsen, J., Evaluating user interface systems research. In Proc. UIST, ACM Press (2007), 251-258.

7. de Kort, Y.A.W. and IJsselsteijn, W.A. People, places, and play: player experience in a socio-spatial context. Computers in Entertainment (CIE), 6 (2). (2008).

8. Flanagan, M., Nissenbaum, H., Belman, J. and Diamond, J., A method for discovering values in digital games. In Proc. DiGRA 2007, (2007).

9. Fogtmann, M.H., Fritsch, J. and Kortbek, K.J., Kinesthetic Interaction - Revealing the Bodily Potential in Interaction Design. In Proc. OZCHI 2008, ACM Press (2008).
10. Halskov, K. and Dalsgaard, P., Inspiration card workshops. In Proc. DIS 2006, ACM Press (2006), 211.

11. Hornecker, E., Creative idea exploration within the structure of a guiding framework: the card brainstorming game. In Proc. TEI 2010, ACM Press (2010), 101-108.

12. Landay, J.A. I give up on CHI/UIST, 2009. http://dubfuture.blogspot.com/2009/11/i-give-up-onchiuist.html.

13. Lanningham-Foster, L., Jensen, T.B., Foster, R.C., Redmond, A.B., Walker, B.A., Heinz, D. and Levine, J.A. Energy Expenditure of Sedentary Screen Time Compared With Active Screen Time for Children. Pediatrics, 118 (6). (2006), 1831-1835.

14. Lin, J., Mamykina, L., Lindtner, S., Delajoux, G. and Strub, H., Fish'n'Steps: Encouraging Physical Activity with an Interactive Computer Game. In Proc. UbiComp 2006, ACM Press (2006), 261-278.

15. Lucero, A. and Arrasvuori, J., PLEX Cards: a source of inspiration when designing for playfulness. In Proc. Fun and Games 2010, ACM Press(2010), 28-37.

16. Mokka, S., Väätänen, A., Heinilä, J. and Välkkynen, P., Fitness computer game with a bodily user interface. In Proc. Entertainment Computing, 2003, 1-3.

17. Mueller, F., Agamanolis, S., Gibbs, M. and Vetere, F., Remote Impact: Shadowboxing over a Distance. In Proc. CHI 2009, ACM Press (2009), 3531-3532.

18. Mueller, F., Agamanolis, S. and Picard, R., Exertion Interfaces: Sports over a Distance for Social Bonding and Fun. In SIGCHI conference on Human factors in computing systems, 2003,ACM, 561-568.

19. Mueller, F., Edge, D., Vetere, F., Gibbs, M.R., Agamanolis, S., Bongers, B. and Sheridan, J.G., Designing Sports: A Framework for Exertion Games. In Proc. CHI 2011, ACM Press (2011), 2651-2660.

20. Mueller, F., Gibbs, M. and Vetere, F., Design Influence on Social Play in Distributed Exertion Games. In Proc. CHI 2009, ACM Press (2009), 1539-1548.

21. Mueller, F., Vetere, F., Gibbs, M.R., Edge, D., Agamanolis, S. and Sheridan, J.G., Jogging over a distance between Europe and Australia. In Proc. UIST 2010, ACM Press (2010), 189-198.

22. Neuman, W.L. Social Research Methods. Pearson Education, USA, 2006.

23. Schell, J. The Art of Game Design - The Cards. http://artofgamedesign.com/cards/.

24. Shah, J.J., Smith, S.M., Vargas-Hernandez, N., Gerkens, D.R. and Wulan, M., Empirical studies of design ideation: Alignment of design experiments with lab experiments. in ASME Design Theory \& Methods Conference, (2003), 2-6.

25. Strauss, A. and Corbin, J. Basics of Qualitative Research: Techniques and Procedures for Developing Grounded Theory. SAGE Publications, Thousand Oaks, CA, USA, 1998. 